\title{
Hemodiafiltración en línea como terapia de reemplazo renal crónica. Primera experiencia nacional en el Hospital Universitario
}

\author{
Ricardo Silvariño, Cecilia Baccino, Patricia Larre Borges, Ana Laura Guerisoli, \\ Verónica Miranda, Paula Búcalo, Francisco González-Martínez, Alejandro Ferreiro, \\ Liliana Gadola, Oscar Noboa
}

\section{Resumen}

La enfermedad renal crónica tiene una prevalencia estimada de 6,5\% a 8\% en los adultos mayores de 18 años en Uruguay. A pesar de los esfuerzos por realizar un diagnóstico temprano y retrasar su progresión un porcentaje de pacientes requiere terapia de reemplazo renal (TRR) mediante diálisis, con una tasa de incidencia anual de 166 pacientes/millón de población. A pesar de las mejoras en el cuidado nefrológico y en las técnicas de hemodiálisis, la mortalidad anual de los pacientes en esta técnica es elevada en nuestro país (16,5\%) y en todo el mundo. Con el objetivo de mejorar estos aspectos se han ensayado técnicas dialíticas que asocian la convección como estrategia para depurar moléculas de mayor tamaño que habitualmente no se depuran en la hemodiálisis convencional. La hemodiafiltración en línea (HDF-OL) es una técnica convectiva. Cuando se utiliza como TRR crónica se asocia a una reducción de la mortalidad de 30\%-35\% comparada con la hemodiálisis convencional. En el año 2014 se instrumentó esta técnica en el Hospital de Clínicas, siendo el centro pionero en el país en contar con ella como TRR crónico. El proceso de implementación implicó cambios de la infraestructura (monitores de diálisis, centro de tratamiento del agua), formación de recursos humanos, cambios en el funcionamiento y controles microbiológicos programados. El control de calidad sistemático y los diferentes estudios realizados en este período de cinco años han mostrado que es una técnica segura, capaz de remover solutos de tamaño medio y de disminuir los requerimientos de eritropoyetina. No se encuentra aún financiada por el sistema de salud, lo que puede constituir una barrera en su difusión a nivel nacional. En el presente trabajo se revisan las características fundamentales de la hemodiafiltración, su beneficio comparado con la hemodiálisis convencional, y el proceso de implementación de la técnica junto con algunos resultados iniciales en el Hospital de Clínicas.

Palabras clave: Diálisis renal

Hemodiafiltración en línea

Mortalidad

Key words: $\quad$ Renal Dialysis

Hemodiafiltration online

Mortality

Centro de Nefrología, Hospital de Clínicas, Universidad de la República, Montevideo, Uruguay

Los autores declaran no tener conflictos de interés.

La totalidad de los proyectos de investigación realizados en el Centro de Nefrología fueron aprobados por el Comité de Ética de la Investigación del Hospital de Clínicas.

Correspondencia: Dr. Oscar Noboa. Centro de Nefrología. Hospital de Clínicas. Universidad de la República. Av. Italia 2870 , piso 14, CP 11600.

Montevideo, Uruguay.

Correo electrónico: onoboa@hc.edu.uy / onoboa@gmail.com

Recibido: 15/8/19

Aprobado: 11/12/19 


\section{Introducción}

El término enfermedad renal crónica (ERC) hace referencia a un conjunto heterogéneo de trastornos que afectan la estructura o función del riñón. Se define por la presencia de alteraciones estructurales (albuminuria, alteraciones del sedimento urinario, alteraciones histológicas en la biopsia renal, alteraciones anatómicas en técnicas de imagen) o funcionales (caída del filtrado glomerular, disfunción tubular, entre otras), mantenidas durante al menos tres meses y con implicancias para la salud ${ }^{(1)}$. Se estima que en Uruguay $6,5 \%$ a $8 \%$ de los adultos mayores de 18 años tiene ERC $^{(2)}$. Como consecuencia de políticas de salud dirigidas al control de enfermedades crónicas no transmisibles, e iniciativas que persiguen el diagnóstico precoz y control de factores de progresión de la ERC, entre las que destaca el Programa de Salud Renal (PSR) ${ }^{(3)}$, solo una minoría de los pacientes requerirá terapias de reemplazo renal (TRR), como lo son el trasplante, la diálisis peritoneal (DP) y la hemodiálisis (HD). Actualmente, reciben TRR (HD + DP) 2.654 pacientes, lo que corresponde a una tasa de prevalencia de 763 pacientes por millón de población (pmp). La tasa incidencia reportada en el último informe del Registro Uruguayo de Diálisis (RUD) fue de 166 pmp (204 pmp en Montevideo, $140 \mathrm{pmp}$ en el interior del país $)^{(4)}$. Uruguay cuenta con acceso universal a la TRR financiada por el Fondo Nacional de Recursos (FNR) y desarrollada en 46 centros ( 38 de hemodiálisis [HD] y 8 de diálisis peritoneal $[\mathrm{DP}])$ distribuidos en el territorio nacional, con mayor presencia en Montevideo (18/38 centros de HD y $7 / 8$ centros de DP) ${ }^{(4)}$. La mayor parte de los pacientes recibe TRR mediante HD, encontrándose al cierre del último informe 328 pacientes en DP, lo que corresponde a $12 \%$ de total de pacientes en $\mathrm{TRR}^{(4)}$. A pesar de la mejora en los cuidados así como el avance en los procedimientos de hemodiálisis, la mortalidad anual no ajustada de los pacientes en TRR permanece alta, $10 \%-22 \%$ en Europa $^{(5)}$ y $16,5 \%$ (no ajustada) en Uruguay $^{(4)}$. Con el objetivo de mejorar la mortalidad en diálisis es que se han explorado las técnicas dialíticas que incorporan la convección como parte del tratamiento. El objetivo de esta revisión es describir las características fundamentales de la hemodiafiltración, su beneficio comparado con la hemodiálisis convencional, y el proceso de implantación de la técnica junto con algunos resultados iniciales en el Hospital de Clínicas.

\section{Hemodiafiltración}

La hemodiálisis es una técnica de depuración extracorpórea. Suple parcialmente alguna de las funciones del riñón: eliminación de solutos (principalmente desechos nitrogenados), eliminación de agua, regulación del equi- librio ácido-base y electrolítico, no siendo eficaz en suplir funciones endócrinas ni metabólicas. Consiste en interponer una membrana semipermeable (fibra o dializador) entre dos compartimentos que circulan a contracorriente: la sangre del paciente y el líquido o baño de diálisis, este último constituido principalmente por agua tratada estéril a la que se adicionan electrolitos y bicarbonato ${ }^{(6)}$. La membrana semipermeable permite el pasaje de agua y solutos de pequeño y mediano peso molecular, pero no proteínas ni células sanguíneas, ya que no son capaces de atravesar los poros de ésta. El principal mecanismo fisicoquímico que regula esta función es la difusión, que es consecuencia del transporte pasivo de solutos a través de la membrana del dializador por diferencia de concentración entre ambos compartimentos (figura 1). Simultáneamente, por gradiente de presión hidrostática, generado y controlado por el monitor de diálisis, entre el circuito sanguíneo y el líquido de diálisis se produce la ultrafiltración de agua y solutos por convección. La convección consiste en el paso simultáneo a través de la membrana de diálisis de agua plasmática acompañada de solutos. Este pasaje de agua y solutos no ocurre de forma pasiva como en la difusión, sino que necesita la generación de un gradiente de presión hidrostática (figura 1). Al líquido extraído de la sangre a través de la membrana por este mecanismo se le llama ultrafiltrado ${ }^{(6)}$. El volumen habitual de ultrafiltrado que se extrae en cada sesión de hemodiálisis debería ser equivalente al volumen ganado por el paciente entre cada procedimiento de hemodiálisis con el objetivo de evitar la sobrehidratación. A diferencia de la difusión, que permite eliminar únicamente moléculas de pequeño tamaño, la convección permite eliminar moléculas de mayor tamaño.

Con el objetivo de aumentar la eliminación de moléculas de mediano y gran tamaño que se transfieren mal por difusión, es que nacen las técnicas convectivas. En ellas la transferencia de solutos se realiza mediante el principio de arrastre por flujo y depende del tamaño de los poros de la membrana semipermeable y de la tasa de ultrafiltración. Generalmente las técnicas convectivas utilizan una membrana de gran permeabilidad y realizan una ultrafiltración muy superior a la necesaria para eliminar el líquido acumulado entre cada sesión de diálisis. Este exceso de volumen que se le extrae al paciente debe ser repuesto por un líquido de sustitución con una composición fisiológica ${ }^{(7)}$. Las técnicas convectivas pueden utilizar de forma exclusiva la convección sin generarse difusión asociada, técnica que se denomina hemofiltración. Si a la convección se asocia difusión, la técnica se denomina hemodiafiltración. Cuando el líquido ultrafiltrado en exceso para lograr mayor convección se repone en base a soluciones de electrolitos administrados al cir- 


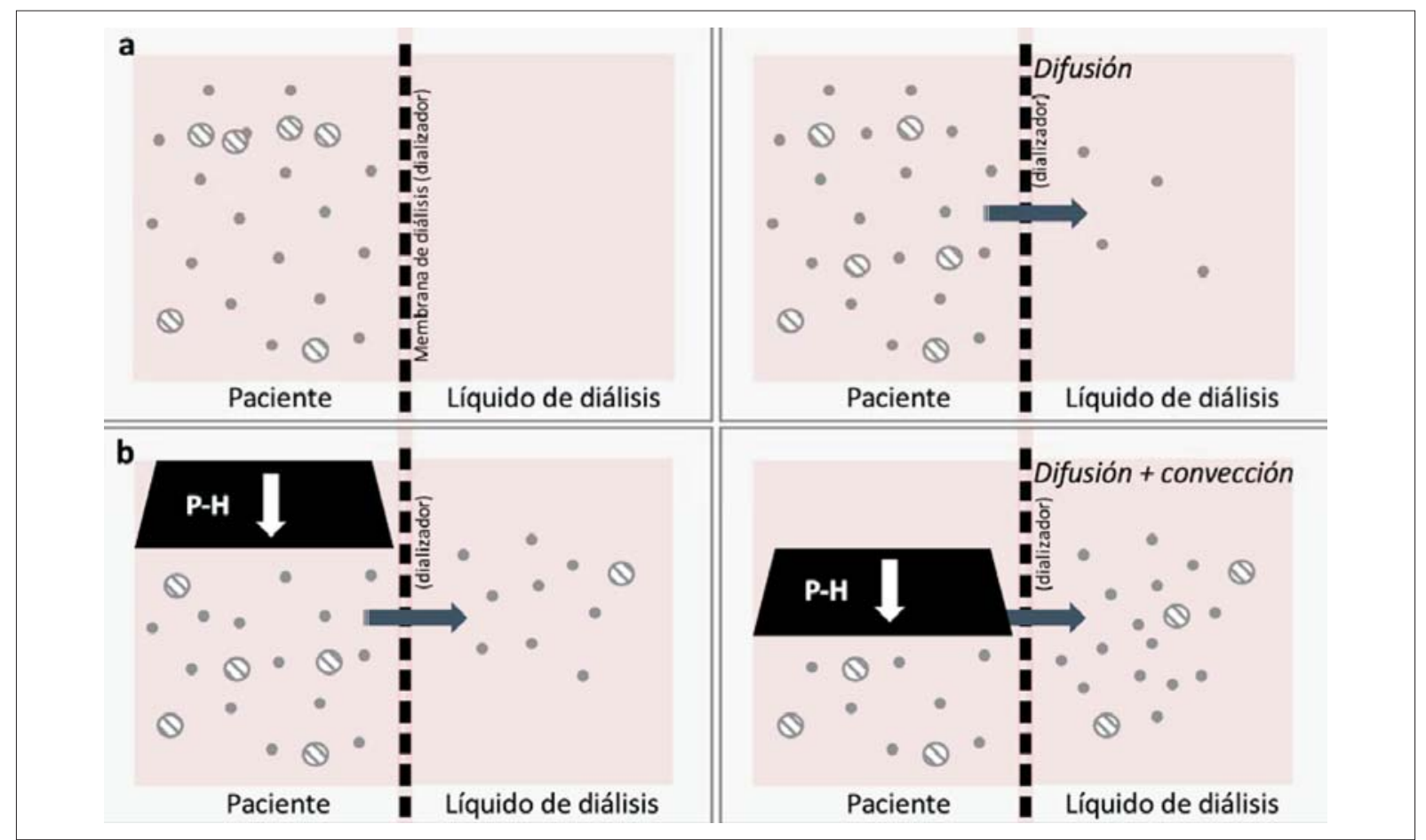

Figura 1. La difusión (a) es el mecanismo mediante el cual los solutos atraviesan una membrana semipermeable de forma pasiva a favor de su gradiente de concentración. En la convección (b) los solutos atraviesan la membrana semipermeable junto con el solvente (agua) de forma activa, a favor de un gradiente de presión hidrostática (P-H). Modificado de ${ }^{(5)}$.

cuito sanguíneo directamente de bolsas preparadas en laboratorios farmacéuticos se denomina "convección clásica". Si la reposición intravenosa al circuito sanguíneo se realiza en base al propio baño de diálisis generado en el monitor, con igual composición que la solución o baño de diálisis, se denomina "convección en línea"(7). La hemodiafiltración en línea combina entonces los principios de difusión y convección, y utiliza el baño de diálisis que se va produciendo durante el procedimiento como líquido de reposición. Para que pueda ser utilizado como sustitución debe ser de alta calidad y reunir las condiciones del denominado "baño ultrapuro". Tiene que ser fabricado con agua ultrapura obtenida con doble sistema de ósmosis inversa, debe de estar en continua recirculación para evitar estancamiento y así riesgo de contaminación, y antes de ser infundido en el circuito sanguíneo debe pasar por al menos dos filtros para endotoxinas intercalados en el circuito de agua y el baño de diálisis. Esta forma de producción permite utilizar grandes volúmenes disponibles para la reposición. El líquido de reposición puede infundirse después de la salida de la sangre de la fibra de diálisis (luego que se ha realizado la convección), técnica que se denomina posdilucional, o, por el contrario, puede infundirse antes de que la sangre entre a la fibra de diálisis (antes de que ocurra la convec- ción), técnica que se denomina predilucional ${ }^{(7)}$ (figura 2). La hemodiafiltración en línea con infusión del líquido de sustitución posdilucional es el procedimiento que consigue los mejores resultados en la depuración de todo tipo de moléculas. La infusión predilucional disminuye problemas derivados de la hemoconcentración, pero este descenso de concentración de solutos como consecuencia de la hemodilución reduce su transferencia por convección ${ }^{(7)}$. Es por este último fenómeno que se recomiendan volúmenes de reposición más elevados cuando la reposición es predilucional.

\section{Beneficio de la hemodiafiltración sobre la hemodiálisis convencional}

La hemodiafiltración en línea (HDF-OL) es una técnica muy difundida, inicialmente en Europa y poco después en Estados Unidos, como alternativa a la hemodiálisis para pacientes que requieren TRR. Es útil tanto en pacientes con una injuria renal aguda como en aquellos bajo tratamiento sustitutivo de forma crónica. Como TRR crónica ha demostrado marcados beneficios que se derivan de la remoción mantenida en el tiempo de moléculas de tamaño mediano y grande, muchas de las cuales están vinculadas a la toxicidad urémica, inflamación crónica, lesión endotelial y desarrollo de daño vascur 

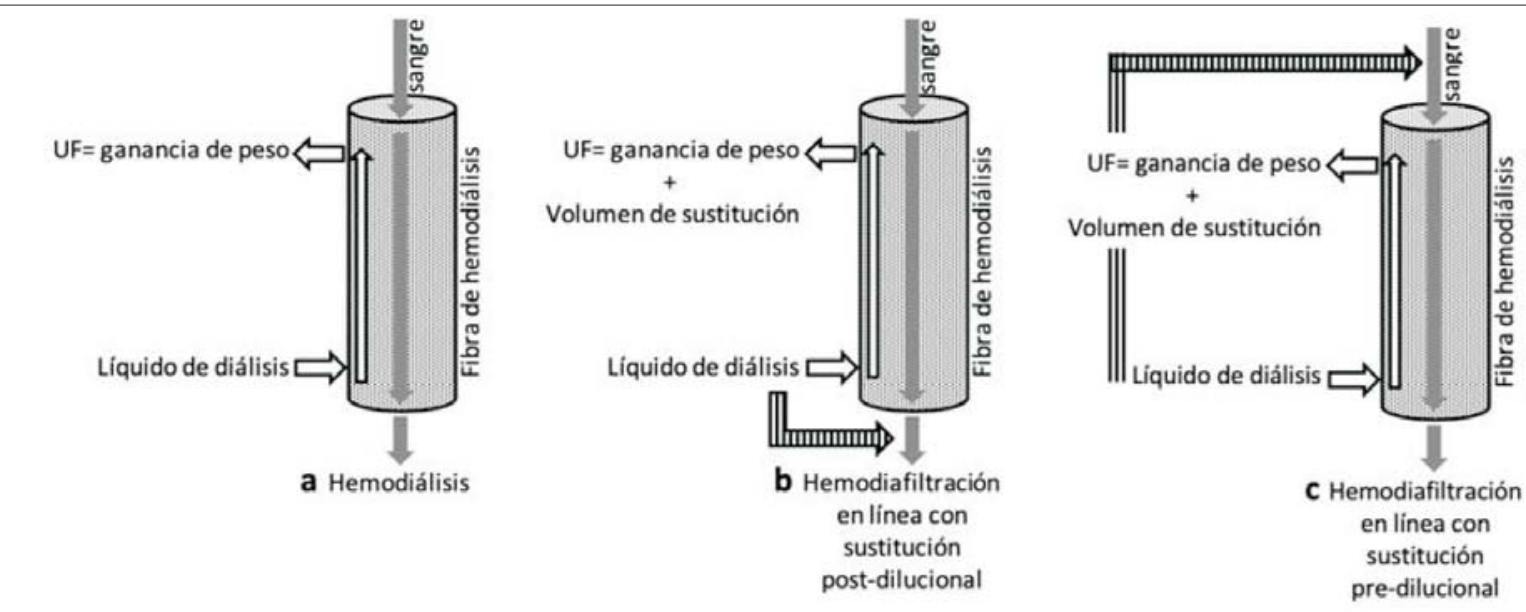

Figura 2. Hemodiálisis (a), la ultrafiltración (UF) se programa en cada sesión según la ganancia de peso del paciente y la valoración del estado de hidratación. Hemodiafiltración en línea con reposición posdilucional (b) y predilucional (c), a la ultrafiltración planificada según peso y valoración del paciente se deben agregar los litros de sustitución infundidos. Modificado de ${ }^{(6)}$.

$\operatorname{lar}^{(8)}$. Maduell y colaboradores ${ }^{(9)}$ aportaron evidencia relevante en esta línea. Se trató de un estudio retrospectivo y multicéntrico en el que incluyeron 906 pacientes bajo TRR mediante hemodiálisis. Asignaron un grupo de 450 pacientes que se mantuvo en la técnica de hemodiálisis crónica y otro grupo de 456 pacientes que recibieron hemodiafiltración en línea con reposición posdilucional. El objetivo primario fue evaluar mortalidad por todas las causas y como objetivo secundario evaluaron mortalidad de causa cardiovascular, hospitalización por todas las causas, tolerabilidad del tratamiento y datos analíticos de laboratorio. Comparado con los pacientes que se mantuvieron en hemodiálisis, el grupo asignado a HDF-OL tuvo una disminución de $30 \%$ de muerte por todas las causas, reducción de riesgo de muerte de causa cardiovascular de $33 \%$, y reducción del riesgo de muerte de causa infecciosa de $55 \%$. El número necesario a tratar (cambio de hemodiálisis a HDL-OL) para evitar una muerte anual fue de 8 . La incidencia de complicaciones como hipotensión vinculada a los procedimientos u hospitalización por todas las causas fue menor en el grupo de HDF-OL. El volumen convectivo promedio por sesión en esta población de estudio fue de 22,9-23,9 $\operatorname{litros}^{(9)}$. En el estudio DOPPS (Dialysis Outcomes and Practice Patterns Study), Canaud y colaboradores ${ }^{(10)}$ compararon la mortalidad de pacientes que recibieron HDF-OL y HD convencional en una larga cohorte de 2.165 pacientes seguidos de forma prospectiva en cinco centros de Europa. Del total de pacientes, $11,7 \%$ recibieron TRR mediante HDF-OL y $88,3 \%$ hemodiálisis convencional. Entre los pacientes que recibieron HDF-OL se consideró de alta eficiencia (4,5\%) aquellos que recibieron un volumen de sustitución de
15-24,9 litros en cada procedimiento y HDF-OL de baja eficiencia $(7,2 \%)$ cuando el volumen de sustitución fue de 5-14,9 litros por procedimiento. Entre quienes recibieron HD convencional, se clasificó según el tipo de dializador utilizado, en HD de alto flujo $(25,2 \%)$ y HD de bajo flujo $(63,1 \%)$. Durante el período de observación (1998-2001) la mortalidad fue 12,8 muertes/100 pacientes/año en el grupo de HD de bajo flujo, 12,7 muertes/100 pacientes/año en el grupo de HD de alto flujo, 12,6 muertes/100 pacientes/año en el grupo HDF de baja eficiencia y 8,9 muertes/100 pacientes/año en el grupo HDF de alta eficiencia. El riesgo relativo de mortalidad, luego de ajustadas múltiples variables, fue 35\% menor en el grupo que recibió HDF-OL de alta eficiencia comparado con el grupo de HD de bajo flujo (RR $0,65, \mathrm{p}=0,01)^{(10)}$. En ambos estudios europeos se evaluó el beneficio de la HDF-OL con sustitución posdilucional. En Japón, donde la HDF-OL ha tenido una reciente difusión, la sustitución se realiza con mayor frecuencia predilucional. En la actualidad se tratan con HDF-OL en ese país 60.000 pacientes, $95 \%$ de los cuales reciben sustitución predilucional. En un reciente estudio japonés ${ }^{(1)}$ se evaluó la mortalidad por todas las causas, a un año de tratamiento, comparando pacientes en hemodiálisis convencional (85.202 pacientes) contra pacientes bajo tratamiento con HDF-OL con sustitución predilucional. Quienes recibieron tratamiento con HDF-OL fueron evaluados en función del volumen de sustitución, identificándose un grupo de pacientes con volúmenes de sustitución mayores a 40 litros/sesión (HDF-OL predilucional con altos volúmenes de sustitución) (2.548 pacientes), y otro con volúmenes de sustitución menores a 40 litros/sesión (HDF-OL predilucional con bajos volúme- 
nes de sustitución) (2.424 pacientes). Luego de ser macheados utilizando la herramienta de propensity score no hubo diferencias significativas en las características basales de los pacientes en HD vs aquellos en HDF-OL. La mortalidad a un año, por todas las causas, fue significativamente menor en el grupo bajo HDF-OL vs HD (HR 0,834, IC95\% 0,705-0,986, $\mathrm{p}=0,029)$. Cuando se comparó entre los grupos de HDF-OL en función del volumen de sustitución, se observó que el grupo con altos volúmenes de sustitución (media 50,3 \pm 10,2 litros/sesión) tuvo una mortalidad a un año, por todas las causas y de causa cardiovascular, significativamente menor que el grupo con bajos volúmenes de sustitución (HR 0,664, IC95\% 0,428-0,968, $\mathrm{p}=0,018$ ).

Si bien la reducción de pequeños solutos es similar en la HDF-OL y en la $\mathrm{HD}^{(12)}$, la capacidad de remoción de toxinas urémicas de tamaño mediano y grande es superior en la HDF-OL de alta eficiencia ${ }^{(13)}$. En los estudios en los que se midió la beta2-microglobulina como molécula de gran tamaño, se objetivó que la remoción de la misma fue $20 \%$ a $30 \%$ superior en la HDF-OL que en la HD convencional de alto flujo $(72,7 \% \text { vs } 49,7 \%)^{(14)}$. Asimismo, el tratamiento regular con HDF-OL redujo el nivel prediálisis de beta2-microglobulina circulante ${ }^{(14)}$. Otros estudios demostraron la capacidad de la HDF-OL para remover moléculas de gran tamaño, como la mioglobina y la proteína ligadora del retinol ${ }^{(15,16)}$. Algunos solutos, por ejemplo, p-cresol, que circulan unidos a proteínas ligadoras y son considerados toxinas urémicas, se remueven más eficientemente con la HDF-OL que con la HD de alto flujo $^{(17)}$. Asimismo, la HDF-OL reduce los niveles circulantes de productos finales de la glicosilación avanzada que se han vinculado a complicaciones a largo plazo en los pacientes en hemodiálisis ${ }^{(18)}$.

La HDF-OL se ha mostrado superior a la HD en mantener estabilidad hemodinámica intradiálisis, mejorando la tolerancia de los procedimientos, principalmente en pacientes añosos y con múltiples comorbilidades ${ }^{(19)}$. Este fenómeno se cree que responde a un incremento en el tono vascular periférico arterial y venoso, asociado al relleno constante del espacio intravascular con el líquido de reposición ${ }^{(20)}$. A esto último se asocia la presencia de sodio en el fluido de reposición, la remoción de mediadores de la vasodilatación y la mejoría de la actividad simpática ${ }^{(21)}$.

El uso de membranas biocompatibles de alta permeabilidad en la HDF-OL reduce la bioactivación y la subsecuente inflamación sistémica ${ }^{(19)}$. Los pacientes bajo tratamiento con HDF-OL tienen menores niveles de mediadores proinflamatorios, como la interleucina 1 , interleucina- 6 y factor de necrosis tumoral ${ }^{(22)}$. Otro beneficio que se ha observado es que los pacientes con HDF-OL presentan niveles más altos de hemoglobina con menores requerimiento de estimulantes de la eritropoyesis que los pacientes bajo HD convencional ${ }^{(23)}$.

En la población pediátrica, la HDF-OL ha mostrado beneficios en disminuir el riesgo cardiovascular y adicionalmente mejorar el crecimiento ponderal de los pacientes. En un estudio reciente, Shroff y colaboradores $^{(24)}$ evaluaron el cambio anual en el espesor íntima-media carotídea y en el score de crecimiento en 190 niños seguidos durante un año provenientes de 28 centros, 78 en tratamiento con HD convencional y 55 bajo HDF-OL. En el grupo bajo HDF-OL el espesor íntima-media carotídea mejoró significativamente a un año de seguimiento, mientras que permaneció sin cambios en el grupo bajo HD convencional $(\mathrm{p}=0,02)$. Asimismo, cuando se evaluó el incremento ponderal se objetivó que el grupo bajo HDF-OL tuvo un cambio anual positivo significativo comparado con el grupo bajo HD convencional, en el que la altura (evaluada mediante height SD score) permaneció incambiada durante el seguimiento $(\mathrm{p}=0,005)$.

\section{Implementación de HDF-OL como terapia de reemplazo renal crónica en el Hospital de Clínicas ${ }^{(25)}$}

\section{1) Equipamiento e insumos}

Monitores de hemodiálisis. Para la implementación de la técnica se requieren equipos de hemodiálisis (monitores) con características particulares. Desde el año 2011, el Centro de Nefrología contaba con los equipos Toray $8000^{\circledR}$, y en el año 2019 se incorporaron dos equipos adicionales Nipro NCU-18 ${ }^{\circledR}$. Ambos equipos cuentan con doble cuerpo de bomba (segunda bomba peristáltica para infusión de líquido de reposición), contador de volumen de sustitución administrado, módulo de balance de fluido (capaz de computar el fluido administrado y determinar la extracción de un volumen igual a la suma de fluido administrado + ultrafiltración programada), y un software de alta precisión desarrollado para esta técnica. Cumplen además con el requerimiento de tener un módulo de generación de líquido de diálisis ultrapuro (primer filtro TET del dializado), módulo de generación de líquido de sustitución (segundo filtro TET de la sustitución) y ultrafiltros/microfiltros intercalados en el circuito hidráulico del monitor compuestos por membranas capilares de fibra hueca de alto flujo que impiden el paso de microorganismos contaminantes y tienen gran capacidad de adsorción de endotoxinas (figura 3).

Dializadores. Se adquirieron fibras de diálisis sintéticas, de alto flujo y parcialmente hidrófilas. Son membranas de alta permeabilidad hidráulica (alto flujo) con un coeficiente de ultrafiltración (Kuf) $>20 \mathrm{ml} / \mathrm{h} / \mathrm{mmHg} / \mathrm{m}^{2}$ (50 $\mathrm{ml} / \mathrm{h} / \mathrm{mmHg}$ ), alta permeabilidad a solutos (porosidad) con 


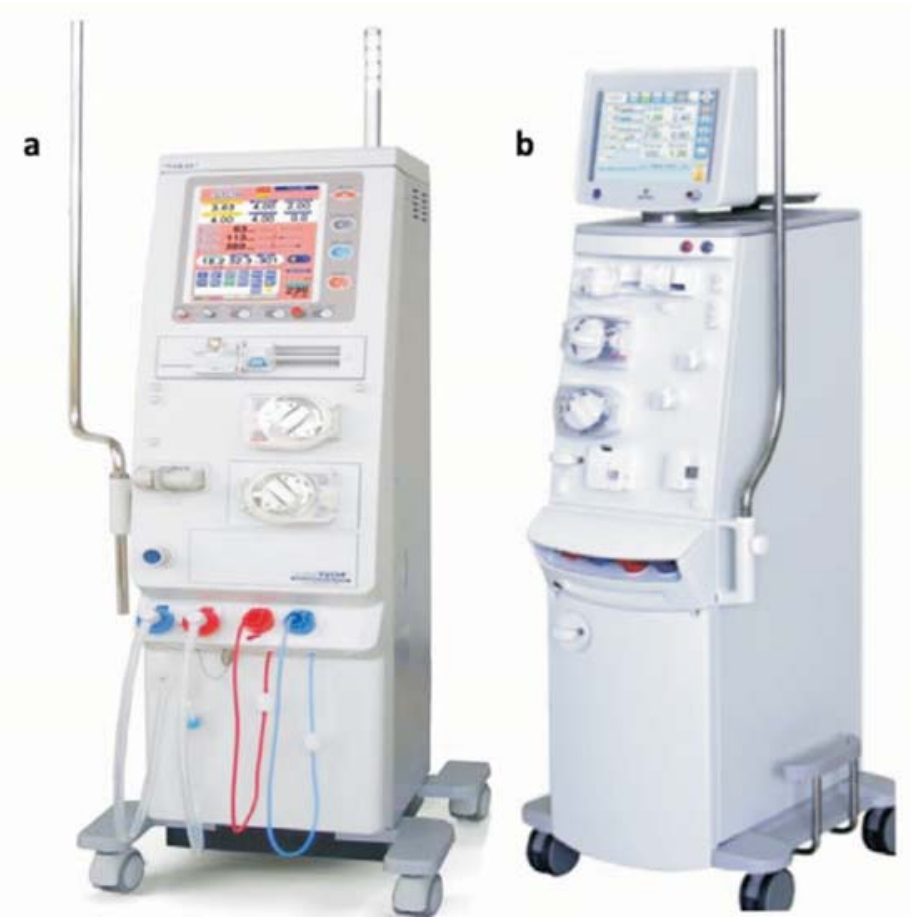

Figura 3. Monitores para HDF-OL: (a) Toray $8000^{\circledR}$, (b) Nipro NCU-18 ${ }^{\circledR}$.

KoA (coeficiente que mide porosidad y espesor de la membrana) de urea $>600$ y un KoA de beta2-microglobulina $>60$, y un área de superficie elevada de entre 1,5 y 2,1 $\mathrm{m}^{2}$. Se definió desde el inicio la no reutilización de las fibras para minimizar al extremo el riesgo infeccioso.

Planta de tratamiento de agua. La HDF-OL requiere un líquido de reposición en línea estéril y apirógeno. El agua "pura" utilizada para hemodiálisis convencional requiere cumplir con el requisito de tener un recuento bacteriano $<100 \mathrm{UFC} / \mathrm{ml}$ y un recuento de endotoxinas $<0,25 \mathrm{UE} / \mathrm{ml}$. El líquido de sustitución para hemodiafiltración en línea requiere un umbral microbiológico más exigente con un recuento bacteriano $<0,1 \mathrm{UFC} / \mathrm{ml}$ y un recuento de endotoxinas $<0,03 \mathrm{UE} / \mathrm{ml}$, bajo estas condiciones se considera agua ultrapura ${ }^{(26)}$. El agua ultrapura se logra con un sistema de tratamiento de agua de alta calidad asociado a los filtros microbiológicos ubicados en los monitores de diálisis. Asimismo, el agua debe cumplir con requisitos fisicoquímicos más exigentes que los requeridos para el agua empleada en hemodiálisis (conductividad medida a $25{ }^{\circ} \mathrm{C}: 1 \mathrm{a} 1,3 \mu \mathrm{S} / \mathrm{cm}-1, \mathrm{pH}$ entre 5,0 y 7,0 , carbono orgánico total (TOC) $0,5 \mathrm{mg} / 1$ y nitratos maiximo $0,2 \mathrm{mg} / \mathrm{L})^{(26)}$. Para mejorar el tratamiento del agua se implementaron cambios en el Hospital de Clínicas consistentes en optimizar el sistema de pretratamiento (se instaló filtro de zeolita y sistema de meta-bisulfito) y el sistema de tratamiento (se instaló una ósmosis inversa de doble paso).
Distribución del agua ultrapura. En 2012, se instaló un circuito loop de polietileno para transporte de agua ultrapura que se utilizó hasta 2019 de forma exclusiva para HDF-OL. En 2019, se instaló un loop de polietileno biocompatible que provee de agua ultrapura a la totalidad de equipos de la sala de hemodiálisis.

Controles microbiológicos: se incrementó el número de controles microbiológicos según se recomienda en los estándares nacionales e internacionales ${ }^{(26)}$.

\section{2) Formación de recursos humanos}

Durante el período 2012 a 2014 se realizaron instancias de formación teórico-prácticas dirigidas al personal médico y de enfermería. Estas instancias se centraron es aspectos teóricos vinculados a la técnica y en aspectos prácticos vinculados a la selección de los pacientes, así como aspectos operativos del procedimiento.

\section{Resultados de la HDF-OL durante los primeros cinco años de implantación de la técnica}

\section{1) Pacientes}

El primer procedimiento de HDF-OL se realizó en junio de 2014. Desde entonces se trató por un período $\geq 6$ meses un total de 28 pacientes. Al 31 de diciembre de 2018, la población prevalente tratada con HDF-OL fue de 16 pacientes, 11 (69\%) de sexo femenino, todos provenían de la técnica de hemodiálisis crónica. El criterio 
inicial de selección de pacientes candidatos a recibir HDF-OL fue: (1) contar con una fístula arterio-venosa que permitiese un flujo de bomba próximo a $400 \mathrm{ml} / \mathrm{mi}-$ nuto; (2) aceptar el procedimiento, y (3) consentir el inicio de la técnica luego de ser adecuadamente informado. La mediana edad fue de 50 años (42-71) y las nefropatías prevalentes fueron nefropatía tubulointersticial crónica $(8 / 16)$, diabética $(2 / 16)$, poliquistosis renal $(2 / 16)$ y otras (glomerulopatía, enfermedad de Alport, riñón del mieloma, anéfrico por cirugía). El índice de Charlson (comorbilidades) de la población prevalente fue de 5,6. El 100\% de los pacientes cumplió con el tiempo estipulado de diálisis $(12 \mathrm{~h} / \mathrm{semana}$ en tres sesiones), en los 15 pacientes en que la sustitución indicada fue posdilucional se logró un volumen $\geq 24$ litros/procedimiento y en el paciente en que la sustitución fue predilucional la media de volumen fue de 38 litros/procedimiento. En referencia a los accesos vasculares, 10/16 tenían fístula arterio-venosa nativa, 4/16 fístula arteriovenosa protésica y 2/16 catéter de Tessio Cannaud. No se registraron reacciones pirogénicas intradiálisis y no hubo fallecidos en ese período. En referencia a los restantes pacientes que se trataron bajo esta modalidad por un período $\geq 6$ meses: $1 / 12$ falleció en el transcurso del año 2017; 3/12 recibieron un trasplante renal, y los 8/12 restantes salieron transitoria (no permaneciendo un período $\geq 6$ meses) o definitivamente de la técnica de HDF-OL por complicaciones de los angioaccesos (trombosis principalmente), o por incumplimiento en la asistencia que impedía llegar al objetivo del volumen de reposición establecido.

\section{2) Seguridad}

El análisis de los registros sistemáticos del sistema de control de calidad del Centro de Hemodiálisis evidencia que no se observó ninguna complicación grave vinculada a la técnica durante este tiempo. No se registró durante este período ninguna reacción pirogénica intradiálisis en los pacientes que se trataron con esta modalidad, así como tampoco episodios de hipotensión severa sintomática.

\section{3) Depuración de moléculas de tamaño medio}

Además del control sistemático, y con el objetivo de evaluar la eficacia de la HDF-OL para depurar moléculas de tamaño medio comparada con la HD convencional, en 2016 se realizó un trabajo prospectivo ${ }^{(27)}$. Se comparó un grupo de pacientes (grupo 1) tratados mediante HDF-OL durante un período de $7 \pm 4,6$ meses, con un grupo de pacientes (grupo 2) tratado mediante HD convencional durante un período de 54,5 \pm 9,2 meses. El grupo 1 estaba formado por siete pacientes (cinco de sexo masculino) con una edad de 59,4 \pm 12 años, en la totalidad de los pacientes el volumen de reposición fue de 24 litros/sesión. El grupo 2 estaba formado por seis pacientes (tres de sexo masculino) con una edad de $50,3 \pm 11,4$ años. En ambos grupos se midió en plasma: urea, creatinina, beta2-microblobulina, fósforo, calcio, albúmina, vitamina $\mathrm{D}$, transferrina y proteína $\mathrm{C}$ reactiva. Las determinaciones se hicieron: al momento del inicio de la HDF-OL (grupo 1) y en las últimas seis sesiones de cada técnica (determinación pre y postsesión) en ambos grupos. Se obtuvieron 47 dosificaciones pre y postsesión en el grupo 1 y 14 determinaciones en el grupo 2. Se observó un descenso significativo de la transferrina $(p<0,005)$ y de la beta2-microglobulina $(p<0,005)$ en el grupo tratado con HDF-OL (grupo 1). En el grupo 1 se compararon los niveles de beta2-microglobulina entre el tiempo 0 (previo al primer procedimiento de HDF-OL) y el final del seguimiento (niveles presesión previo al cierre del estudio), objetivándose un descenso significativo de $31,4 \pm 13,4$ a $23,1 \pm 11,4 \mu \mathrm{g} / \mathrm{l}(\mathrm{p}<0,05)$. No hubo diferencia significativa entre grupos en el descenso del resto de las moléculas medidas. No hubo complicaciones vinculadas al procedimiento. Este primer estudio permitió objetivar que la HDF-OL logró en nuestra población una depuración significativa de beta2-microglobulina, que es un marcador de depuración de moléculas de tamaño medio. Este descenso fue significativo para cada sesión de HDF-OL y evolutivo en el tiempo ${ }^{(27)}$.

\section{4) Requerimiento de eritropoyetina}

En 2018, con el objetivo de describir los resultados clínicos y analíticos de los pacientes tratados con HDF-OL, se realizó un estudio retrospectivo ${ }^{(28)}$. Fueron incluidos pacientes que recibieron tratamiento mediante HDF-OL durante un tiempo mayor o igual a seis meses en el período 2014-2018. Se mantuvo el criterio inicial de selección de pacientes candidatos a recibir HDF-OL: (1) contar con una fístula arterio-venosa que permitiese un flujo de bomba próximo a $400 \mathrm{ml} / \mathrm{minu}-$ to; (2) aceptar el procedimiento, y (3) consentir el inicio de la técnica luego de ser adecuadamente informado. Se registraron datos patronímicos (edad, sexo), clínicos (cifras de presión arterial pre y posprocedimiento, peso seco, ganancia de peso interdialítica, registro de reacción pirogénica intradiálisis), analíticos (hemoglobinemia, ferritina, fosfatemia, calcemia, albuminemia) y vinculados al tratamiento (requerimiento de eritropoyetina, logro del objetivo terapéutico en el volumen de reposición). La mediana de resultados analíticos de laboratorio fue comparada con el grupo de pacientes prevalentes en el Centro de Hemodiálisis al momento del cierre del estudio. Se obtuvo la totalidad de datos de 17 pacientes (diez de sexo masculino) con 
una edad de $62,5 \pm 2,2$ años y un promedio de 12,99 (11-14) procedimientos/mes. Se comparó con el grupo de pacientes prevalentes en hemodiálisis convencional los que recibieron en promedio 12,99 (12-13) procedimientos/mes. No hubo diferencias significativas entre grupos en lo que refiere a edad, sexo, peso seco estimado, ganancia interdialítica y valores de presión arterial diastólica, sistólica y media pre y posprocedimiento. En referencia a los datos analíticos no hubo diferencia significativa entre grupos en valores de hemoglobinemia, ferritina, fosforemia, calcemia y albúmina. Se objetivó un requerimiento significativamente menor de eritropoyetina ( 18.769 vs $15.578 \mathrm{UI} / \mathrm{mes}) \mathrm{p}<0,05$ en el grupo bajo tratamiento con HDF-OL. La totalidad de los pacientes bajo HDF-OL recibió un volumen de sustitución mayor o igual a 24 1/sesión y no se registraron reacciones pirogénicas intradiálisis en el período evaluado. Este trabajo permitió objetivar que los pacientes en HDF-OL tuvieron un requerimiento menor de eritropoyetina. Una vez más el procedimiento fue seguro y no se registraron complicaciones. Los autores plantearon que una de las posibles causas que explique la ausencia de diferencias en los parámetros bioquímicos entre ambos grupos puede ser un sesgo en la selección de los pacientes para HDF-OL, ya que en múltiples ocasiones son transferidos a esta técnica pacientes con hiperfosfatemias más severas o menor tolerancia hemodinámica a los procedimientos de HD convencional $^{(28)}$.

\section{Financiación del procedimiento}

En Uruguay la totalidad de los procedimientos de diálisis crónica son financiados por el Fondo Nacional de Recursos (FNR). Según un cálculo realizado en el Centro de Nefrología del Hospital de Clínicas, los procedimientos de hemodiafiltración conllevan un incremento del costo próximo a un $30 \%$ adicional al costo de la hemodiálisis. Este incremento en el costo se vincula al uso y descarte de dializadores y circuitos de HDF más costosos, mantenimiento del sistema de tratamiento de agua ultrapura y un requerimiento de mayores controles microbiológicos del agua tratada, entre otros factores. No se incluyó en la estimación de costos los derivados de la adquisición de equipos con doble cuerpo de bomba ni la formación de recursos humanos para realizar la técnica. Actualmente, el FNR no ha generado un arancel diferencial para la HDF-OL, lo que puede ser una barrera en la expansión de la técnica. Múltiples autores coinciden en que la HDF-OL es posiblemente costo-efectivo, con un costo operativo que no supera la HD convencional con membranas de alto flujo ${ }^{(8)}$. A la luz de la nueva evidencia, la difusión de su utilización en otras partes del mundo, y la experiencia nacional positiva descrita, los autores consideran que sería de beneficio poder extender la técnica en nuestro país.

\section{Conclusiones}

La HDF-OL es una técnica dialítica difundida en todo el mundo. Ha demostrado disminuir la mortalidad de pacientes en TRR crónica cuando se compara con la HD convencional. En nuestro país se implantó como TRR crónica en el Hospital de Clínicas, constituyendo esta la primera experiencia a nivel nacional. El número de pacientes bajo tratamiento, así como el tiempo de seguimiento, no permiten sacar conclusiones aún sobre su impacto en la mortalidad. Los controles de calidad sistemáticos del Centro de Nefrología y los estudios iniciales mostraron que es una técnica segura, eficaz en depurar solutos de tamaño medio y en disminuir los requerimientos de eritropoyetina comparado con la HD convencional. El costo es algo superior a la HD y la diferencia no está financiada aún por el sistema de salud, lo que es una barrera para su desarrollo.

\section{Abstract}

Chronic kidney disease has an estimated prevalence of $6.5 \%$ to $8 \%$ in adults older than 18 years old in Uruguay. Despite efforts to make an early diagnosis and delay its progression, a percentage of patients require renal replacement therapy (RRT) with dialysis, the annual incidence rate being 166 patients per million population. Regardless of improvements in nephrology care and hemodialysis techniques, annual mortality ratex for this technique is high in our country $(16.5 \%)$ and around the world. In order to improve these aspects, different dialysis techniques associating convection as a strategy to purify larger molecules that are rarely purified in conventional hemodialysis have been tried out. Online haemodiafiltration (OL-HDF) is a convective technique. When used as a chronic RRT it is associated to a $30-35 \%$ reduction in mortality compared to conventional hemodialysis. In 2014 this technique was introduced in the University Hospital, being it the first center that offered it as chronic renal replacement therapy. The implementation process implied changes in infrastructure (dialysis computer screens, water treatment center), the training of human resources, changes in the operation system and programmed microbiological controls.

A systematic quality control and the different studies conducted in this 5-year period have proved it is a safe technique that removes average size solutes and reduces the erythropoietin requirements. This technique is still not funded by the health system, what may result in an obstacle for it to be applied nationally. 
This study reviews the main features of haemodiafiltration, its benefits when compared to conventional hemodialysis and the process needed to implement the technique, along with initial results in the University Hospital.

\section{Resumo}

No Uruguai a doença renal crônica tem uma prevalência estimada de 6.5 a $8 \%$ nos adultos maiores de 18 anos. Apesar dos esforços para realizar um diagnóstico precoce e retardar sua progressão uma porcentagem de pacientes requer terapia de substituição da função renal (TSFR) mediante diálise, com uma taxa de incidência anual de 166 pacientes/milhão de habitantes. Independentemente das melhorias na atenção nefrológica e nas técnicas de hemodiálise, a mortalidade anual dos pacientes em tratamento com esta técnica é elevada no Uruguai (16.5\%) e no mundo todo. Buscando melhorar esses aspectos foram ensaiadas varias técnicas dialíticas que associam a convecção como estratégia para depurar moléculas de maior tamanho que habitualmente não são depuradas na hemodiálise convencional. A hemodiafiltração on line (HDF-OL) é uma técnica convectiva. Quando é utilizada como TSFR crônica está associada a uma redução da mortalidade de 30-35\% comparada com a hemodiálise convencional. Esta técnica foi instrumentada em 2014 no Hospital de Clínicas, sendo este o centro pioneiro no Uruguai em utilizá-la como TSFR crônico. O processo de implementação impôs mudanças na infraestrutura (monitores de diálise, centro de tratamento da água), formação de Recursos Humanos e mudanças no funcionamento e controles microbiológicos programados. O controle de qualidade sistemático e os diferentes estudos realizados neste período de 5 anos mostraram que é uma técnica segura, capaz de remover solutos de tamanho médio e de reduzir os requerimentos de eritropoietina. A atual falta de financiamento pelo sistema de saúde pode ser uma barreira para sua difusão no país. Neste trabalho faz-se uma revisão das características fundamentais da hemodiafiltração, seu beneficio comparado com a hemodiálise convencional, o processo de implementação da técnica e alguns resultados iniciais do Hospital de Clínicas.

\section{Bibliografía}

1. Kidney Disease Improving Global Outcomes (KDIGO) CKD Work Group. KDIGO 2012 clinical practice guideline for the evaluation and management of chronic kidney disease. Kidney Inter Suppl 2013; 3(1):1-150.

2. Ríos Sarro P, Sola L, Gadola L. Prevalence of CKD in Uruguay. En: García-García G, Agodoa LY, Norris KC, eds. Chronic kidney disease in disadvantaged populations. Lon- don: Elsevier, 2017:35-44. doi: 10.1016/B978-0-12804311-0.00005-4.

3. Schwedt E, Solá L, Ríos P, Mazzuchi N. Improving the management of chronic kidney disease in Uruguay: a National Renal Healthcare Program. Nephron Clin Pract 2010; 114(1):c47-59. doi: 10.1159/000245069.

4. González Bedat C, Ferreiro A, Chifflet L, Ceretta ML. Registro uruguayo de diálisis: informe Anual 2016. Montevideo: Sociedad Uurguaya de Nefrología, 2016.

5. Rayner H, Pisoni R, Bommer J, Canaud B, Hecking E, Locatelli F, et al. Mortality and hospitalization in haemodialysis patients in five European countries: results from the Dialysis Outcomes and Practice Patterns Study (DOPPS). Nephrol Dial Transplant 2004; 19(1):108-20. doi: 10.1093/ ndt/gfg483.

6. Sellarés VL. Hemodiáisis: principios físicos: definiciones y conceptos. En: Lorenzo V, Lòpez Gómez JM, eds. Nefrología al día. Madrid: SEN, 2018:355-68.

7. Fernández M, Teruel JL. Técnicas de hemodiálisis. En: Lorenzo V, López Gómez JM, eds. Nefrología al día. Madrid: SEN, 2018::437-46.

8. Blankestijn P, Grooteman M, Nube M, Bots M. Clinical evidence on haemodiafiltration. Nephrol Dial Transplant 2018; 33(suppl 3):iii53-iii58. doi: 10.1093/ndt/gfy218.

9. Maduell F, Moreso F, Pons M, Ramos R, Mora-Macià J, Carreras J, et al. High-efficiency postdilution online hemodiafiltration reduces all-cause mortality in hemodialysis patients. J Am Soc Nephrol 2013; 24(3):487-97. doi: 10.1681/ASN.2012080875.

10. Canaud B, Bragg-Gresham J, Marshall M, Desmeules S, Gillespie B, Depner T, et al. Mortality risk for patients receiving hemodiafiltration versus hemodialysis: European results from the DOPPS. Kidney Int 2006; 69(11):2087-93. doi: 10.1038/sj.ki.5000447.

11. Kikuchi K, Hamano T, Wada A, Nakai S, Masakane I. Predilution online hemodiafiltration is associated with improved survival compared with hemodialysis. Kidney Int 2019; 95(4):929-38. doi: 10.1016/j.kint.2018.10.036.

12. Krieter D, Collins G, Summerton J, Spence E, Moragues H, Canaud B. Mid-dilution on-line haemodiafiltration in a standard dialyser configuration. Nephrol Dial Transplant 2005; 20(1):155-60. doi: 10.1093/ndt/gfh520.

13. Ward R, Schmidt B, Hullin J, Hillebrand G, Samtleben W. A comparison of on-line hemodiafiltration and high-flux hemodialysis: a prospective clinical study. J Am Soc Nephrol 2000; 11(12):2344-50.

14. Wizemann V, Lotz C, Techert F, Uthoff S. On-line haemodiafiltration versus low-flux haemodialysis. A prospective randomized study. Nephrol Dial Transplant 2000; 15(Suppl 1):43-8. doi: 10.1093/oxfordjournals.ndt.a027963.

15. Maduell F, Navarro V, Torregrosa E, Rius A, Dicenta F, Cruz M, et al. Change from three times a week on-line hemodiafiltration to short daily on-line hemodiafiltration. Kidney Int 2003; 64(1):305-13. doi: 10.1046/j.1523-1755.2003.00043.x. 
16. Maduell F, Navarro V, Cruz M, Torregrosa E, Garcia D, Simon V, et al. Osteocalcin and myoglobin removal in on-line hemodiafiltration versus low- and high-flux hemodialysis. Am J Kidney Dis 2002; 40(3):582-9. doi: 10.1053/ajkd.2002.34918.

17. Bammens B, Evenepoel P, Verbeke K, Vanrenterghem Y. Removal of the protein-bound solute p-cresol by convective transport: a randomized crossover study. Am J Kidney Dis 2004; 44(2):278-85. doi: 10.1053/j.ajkd.2004.04.033.

18. Gerdemann A, Wagner Z, Solf A, Bahner U, Heidland A, Vienken J, et al. Plasma levels of advanced glycation end products during haemodialysis, haemodiafiltration and haemofiltration: potential importance of dialysate quality. Nephrol Dial Transplant 2002; 17(6):1045-9. doi: 10.1093/ ndt/17.6.1045.

19. Canaud B, Wizemann V, Pizzarelli F, Greenwood R, Schultze G, Weber C, et al. Cellular interleukin-1 receptor antagonist production in patients receiving on-line haemodiafiltration therapy. Nephrol Dial Transplant 2001; 16(11):2181-7. doi: 10.1093/ndt/16.11.2181.

20. Donauer J, Schweiger C, Rumberger B, Krumme B, Böhler J. Reduction of hypotensive side effects during online-haemodiafiltration and low temperature haemodialysis. Nephrol Dial Transplant 2003; 18(8):1616-22. doi: 10.1093/ ndt/gfg206

21. Bégin V, Déziel C, Madore F. Biofeedback regulation of ultrafiltration and dialysate conductivity for the prevention of hypotension during hemodialysis. ASAIO J 2002; 48(3):312-5. doi: 10.1097/00002480-200205000-00018.

22. Guth H, Gruska S, Kraatz G. On-line production of ultrapure substitution fluid reduces TNF-alpha- and IL-6 release in patients on hemodiafiltration therapy. Int J Artif Organs 2003; 26(3):181-7. doi: 10.1177/039139880302600301.

23. Maduell F, del Pozo C, García H, Sánchez L, Hdez-Jaras J, Albero M, et al. Change from conventional haemodiafiltration to on-line haemodiafiltration. Nephrol Dial Transplant 1999; 14(5):1202-7. doi: 10.1093/ndt/14.5.1202.

24. Shroff R, Smith C, Ranchin B, Bayazit A, Stefanidis C, Askiti V, et al. Effects of hemodiafiltration versus conventional hemodialysis in children with ESKD: the HDF, heart and height study. J Am Soc Nephrol 2019; 30(4):678-691. doi: 10.1681/ASN.2018100990

25. Baccino C, Larre Borges P, Alvarez D, Phillips C, González F, Noboa O, et al. Desarrollo de la hemodiafiltración en línea en el Centro de Nefrología del Hospital de Clínicas: técnica de terapia de sustitución de la función renal. En: X Congreso Uruguayo de Nefrología: libro de resúmenes. Montevideo: Sociedad Uruguaya de Nefrología, 2016:16.

26. Blanco J, Castillo T, Chifflet L, Leiva G, Nin M, Opertti A, et al. Guía de gestión de calidad del agua para diálisis. 2 ed. Montevideo: FNR, 2014.

27. Larre Borges P, Zulberti C, Villegas N, Sarantes R, Ferrari S, Luzardo L, et al. Aumento de la depuración de moléculas medias con hemodiafiltración on line en al paciente crónico. En: X Congreso Uruguayo de Nefrología: libro de resúmenes. Montevideo: Sociedad Uruguaya de Nefrología, 2016:14

28. Flores R, Guerisoli A, Yosi C, et al. Hemodiafiltracion en linea crónica: seguimiento de los primeros 17 pacientes en Uruguay. En: XI Congreso Uruguayo de Nefrología y V Jornadas Rioplatenses de Nefrología: libro de resúmenes. Montevideo: Sociedad Uruguaya de Nefrología, 2018:31.

\section{Contribución de autores}

Ricardo Silvariño, https://orcid.org/0000-0002-0416-2694. Concepción, diseño, recolección de datos, análisis, redacción, revisión. Cecilia Baccino, https://orcid.org/0000-0001-8029-0172. Concepción, diseño, recolección de datos, análisis, redacción, revisión.

Patricia Larre Borges, https://orcid.org/0000-0001-7857-6462. Revisión.

Ana Laura Guerisoli, https://orcid.org/0000-0002-2187-4300. Revisión.

Verónica Miranda, https://orcid.org/0000-0002-5166-4515. Revisión.

Paula Búcalo, https://orcid.org/0000-0003-0857-9523. Revisión.

Francisco González-Martínez, https://orcid.org/0000-0002-9882-7449. Revisión.

Alejandro Ferreiro, https://orcid.org/0000-0001-6819-8058. Revisión.

Liliana Gadola, https://orcid.org/0000-0001-5041-4760. Revisión.

Oscar Noboa, https://orcid.org/0000-0002-0975-9924. Análisis, redacción, revisión. 